\title{
An Online Annotation Assistant for Argument Schemes
}

\author{
John Lawrence \\ Jacky Visser \\ Chris Reed \\ Centre for Argument Technology \\ University of Dundee, UK \\ j.lawrence/j.visser/c.a.reed@dundee.ac.uk
}

\begin{abstract}
Understanding the inferential principles underpinning an argument is essential to the proper interpretation and evaluation of persuasive discourse. Argument schemes capture the conventional patterns of reasoning appealed to in persuasion. The empirical study of these patterns relies on the availability of data about the actual use of argumentation in communicative practice. Annotated corpora of argument schemes, however, are scarce, small, and unrepresentative. Aiming to address this issue, we present one step in the development of improved datasets by integrating the Argument Scheme Key - a novel annotation method based on one of the most popular typologies of argument schemes - into the widely used OVA software for argument analysis.
\end{abstract}

\section{Introduction}

In argumentative discourse, a speaker or writer intends to convince their audience of a contested point of view (van Eemeren et al., 2014). To convince their audience, an appeal is made to reasoning, either in direct conversation (such as a courtroom discussion), or in indirect or monological settings (such as a political speech). The argumentative quality of such discourse can be evaluated from various perspectives. In the current paper, we focus on the argumentative quality in terms of the acceptability of the reasoning appealed to in the arguments - thus disregarding, e.g., the rhetorical effectiveness, another dimension of the quality of argumentative discourse.

Consider Hillary Clinton's argument in Example (1) - taken from the US2016 annotated corpus of television debates in the lead-up to the 2016 US presidential elections (Visser et al., 2019a). Anticipating that her first asserted proposition might not be outright acceptable to the entire audience, she provides a reason in support. By defending her policy proposal by comparing the dangers of potential terrorists flying to the dangers of them buying guns, Clinton's argument relies on a conventionalised reasoning pattern: that comparable situations should be dealt with similarly.

(1) Hillary Clinton: And we finally need to pass a prohibition on anyone who's on the terrorist watch list from being able to buy a gun in our country. If you're too dangerous to fly, you are too dangerous to buy a gun.

Evaluating an argument begins by identifying the reasoning pattern it is based on. These common reasoning patterns are conceptualised within the field of argumentation theory as 'argument schemes' (Section 2). While corpus-linguistic approaches have gained traction in the study of argumentation - partly motivated by the rise of 'argument mining' (Stede and Schneider, 2018) - these have generally focused on aspects of argumentative discourse other than argument schemes (such as the use of rhetorical figures of speech (Harris and Di Marco, 2017)). The empirical study of argument schemes would greatly benefit from quantitative data in the form of annotated text corpora. Existing corpora annotated with argument schemes, however, tend to be based on restricted typologies, be of limited size, or suffer from poor validation (Section 3).

In the current paper, we aim to support the annotation of argument schemes by combining a recently developed annotation method for one of the leading typologies of argument schemes (Section 4) and a popular online software tool for annotating argumentative discourse, OVA (Section 5). The standard version of OVA, and other software for manual argument annotation, such as Araucaria (Reed and Rowe, 2004), Rationale 


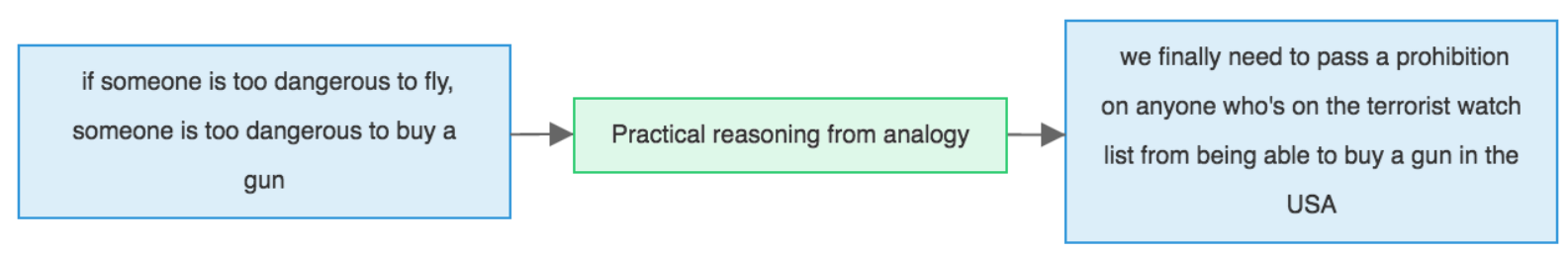

Figure 1: OVA visualisation of the practical reasoning from analogy scheme in Example (1).

(van Gelder, 2007), and Carneades (Gordon et al., 2007) allow the analyst to label arguments with a particular scheme, but they do not offer support to the analyst in the actual scheme selection, which is what our OVA extension is aimed at.

\section{Argument Schemes}

Argument schemes are theoretical abstractions of the conventional patterns of reasoning appealed to in persuasive communication, substantiating the inferential relation between premise(s) and conclusion. The defeasibility of the schemes sets them apart from the strict reasoning patterns of classical formal logic (e.g., Modus Ponens). The type of argument scheme determines its evaluation criteria, commonly expressed as critical questions - owing to the dialectical origins of the notion (van Eemeren and Garssen, 2019). Adequately arguing for a standpoint implies both that the premise(s) of the argument should be acceptable, and that the argumentative connection between the premise(s) and the conclusion can withstand the critical questioning.

Since their introduction (Hastings, 1963; Perelman and Olbrechts-Tyteca, 1969; van Eemeren et al., 1978), argument schemes have become a central topic in argumentation studies, leading to a variety of typologies, e.g., by Schellens (1985), Kienpointner (1992), van Eemeren and Grootendorst (1992), and Walton (1996). The latter has found particular uptake in computation-oriented approaches (Rahwan and Simari, 2009; Baroni et al., 2018), and is the starting point for the annotation tool we currently present.

Walton's typology comprises a great variety of schemes, conventionally occurring in argumentative practices ranging from colloquial discussion to legal adjudication (Walton et al., 2008). Many of the schemes are commonly distinguished in dialectical or informal-logical approaches to argumentation (e.g. argument from sign and argument from cause to effect). Others, however, are more exotic or highly specialised (e.g. argument from arbitrariness of a verbal classification), are closer to modes of persuasion in a rhetorical perspective on argumentation (e.g. ethotic argument), or would in other approaches be considered fallacies (e.g. generic ad hominem). The list also includes composite schemes that combine aspects from various schemes into one (e.g. practical reasoning from analogy combining practical reasoning and argument from analogy).

\section{Annotating Argument Schemes}

The annotation of argument schemes comprises the classification of the inferential relations between premises and conclusions of arguments in accordance with a particular typology. Figure 1 shows a diagrammatic visualisation of the argument of Example (1) with in the middle the classification of the argument scheme as an instance of practical reasoning from analogy. While we start from Walton's typology, alternative approaches are also employed for scheme identification: Green (2015) presents ten custom argument schemes for genetics research articles, Musi et al. (2016) explore annotation guidelines on the basis of the Argumentum Model of Topics (Rigotti and Greco, 2019), and Visser et al. (2019b) annotate argument schemes on the basis of the Periodic Table of Arguments (Wagemans, 2016).

Existing annotations on the basis of Walton's typology tend to use a restricted set of scheme types, and struggle to obtain replicable results. For example, Duschl (2007) initially adopts a selection of nine argument schemes described by Walton (1996), for his annotation of transcribed middle-school student interviews about science fair projects. Later, however, he collapses several schemes into four more general classes no longer directly related to particular scheme types. This deviation from Walton's typology appears to be motivated by the need to improve annotation agreement. The validation of the annotation method does not account for chance agreement, by only providing percentage-agreement scores (in- 


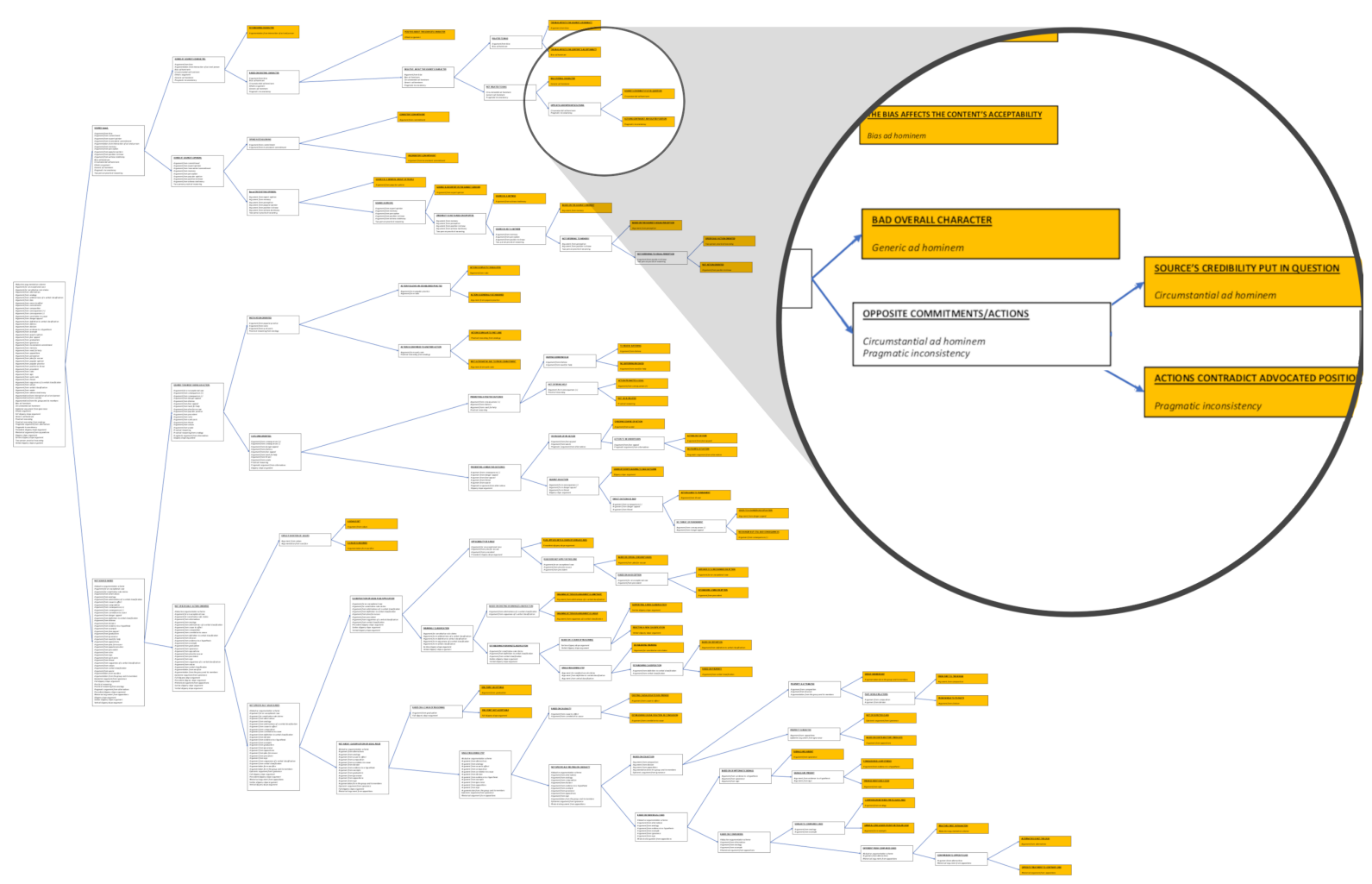

Figure 2: A binary taxonomic tree representation of the ASK

stead of resorting to, e.g., a $\kappa$ or $\alpha$ metric. Out of a total of 17 texts, the inter-annotator agreement is reported on two as $90 \%$ and $84 \%$. No detail is provided on the sampling method.

Similarly, Song et al. (2014) base their annotation on a modification of Walton's typology, settling on a restricted set of three more general schemes: policy, causal, and sample - resulting in Cohen's $\kappa$ scores for inter-annotator agreement ranging from 0.364 to 0.848 . Anthony and Kim (2015) employ a bespoke set of nine coding labels modified from the categories used by Duschl (2007) and nine schemes described in a textbook by Walton (2006). They do not measure any interannotator agreement, opting for a fully open collaborative annotation without any testing of the reliability of the methods.

\section{The Argument Scheme Key (ASK)}

Visser et al. (2018) aim to develop an annotation procedure that stays close to Walton's original typology, while facilitating the reliable annotation of a broad range of argument schemes. The resulting method is reported to yield an interannotator agreement of 0.723 (in terms of Cohen's (1960) $\kappa$ ) on a $10.2 \%$ random sample. The main principle guiding the annotation is the clustering of argument schemes on the basis of intuitively clear features recognisable for annotators. Due to the strong reliance on the distinctive properties of arguments that are characteristic for a particular scheme, the annotation procedure bears a striking resemblance to methods for biological taxonomy the identification of organisms in the various subfields of biology (see, e.g., Voss (1952); Pankhurst (1978)). Drawing on the biological analogue and building on the guidelines used by Visser et al. (2018), we developed a taxonomic key for the identification of argument schemes in accordance with Walton's typology: the Argument Scheme Key - or ASK.

The ASK (reproduced in Appendix A) is a dichotomous identification key that leads the analyst through a series of disjunctive choices based on the distinctive features of a 'species' of argument scheme to the particular type. Starting from the distinction between source-based and other arguments, each further choice in the key leads to either a particular argument scheme or to a further distinction. The distinctive characteristics are numbered, listing between brackets the number of any not directly preceding previous characteristic that led to this particular point in the key. 
In annotating Example (1), an analyst using the ASK follows a sequence of numbered characteristics to identify the argument as an instance of practical reasoning from analogy: 1. Argument does not depend on a source's opinion or character; 17(1). Conclusion is about a course of action; 18. Argument hinges on another motivation for the action [other than its outcome]; 19. Course of action is compared to a similar or alternative action; 21(19). Action is directly compared to another.

The ASK dichotomous identification key can be thought of as a linear textual rendering of a binary taxonomic tree. Figure 2 visualises the decision procedure as such a tree, with each leaf representing an argument scheme label, and all internal nodes showing clusters of schemes that share particular characteristic properties. For each of the numbered binary decision points in the ASK, the tree representation branches into two, thus leading the annotator from the full set of schemes, through their binary choices, to one (and only one) leaf i.e. an argument scheme classification.

\section{The ASK Assistant in the OVA Tool for Argument Annotation}

The Online Visualisation of Argument (OVA) tool (Janier et al., 2014) is a web browser based application (http: / / ova. arg.tech) used by over 3,000 individuals in 38 countries, to analyse and annotate the argumentative structure of natural language text, in contexts ranging from online discussions (Lawrence et al., 2017) to election debates (Visser et al., 2019a). OVA builds on the Argument Interchange Format (AIF) (Chesñevar et al., 2006), an ontology for representing argument analyses compliant with Sematic Web and Linked Data standards, and available in a variety of 'reifications' in languages including JSON, RDF, and Prolog. The software offers import and export of AIF resources from AIFdb (Lawrence et al., 2012), the largest openly available collection of analysed argument, containing over $1.8 \mathrm{~m}$ words and 170,000 claims in more than 15,000 AIF argument maps.

AIF analyses are graphs comprising nodes of information (I-nodes), and instances of schemes (S-nodes); with sub-types of S-nodes representing the application of rules of inference (RA-nodes), and rules of conflict (CA-nodes). An analysis in OVA begins with segmentation by selecting spans of text corresponding to propositions or Argumen- tative Discourse Units (Peldszus and Stede, 2013), and adding these to the canvas as I-nodes. Pairs of I-nodes can then be connected, through RA- or CA-nodes to form structures like that of Figure 1. Complex argumentation structures (Groarke et al., 1997; Snoeck Henkemans, 1992) can, in turn, be formed by connecting an I-node to an existing Snode, or by chaining the connections.

Whilst the original version of OVA allows for a user to label any RA-node as an instance of an argument scheme from Walton's typology by selecting from a dropdown list, in this work, we have introduced the option for users to be guided through this process using the ASK. In order to achieve this, the ASK is first converted into JSON , a fragment of which is shown in Listing 1 (we have also made the full JSON representation available online ${ }^{1}$ for download and integration into other argumentation tools). Each branching point in the ASK has two options, which are represented by their text, and a result - where the result can either be a scheme name ("resulttype": "scheme") or a pointer to another branching point ("resulttype": "branch").

Listing 1: A fragment of the ASK in JSON

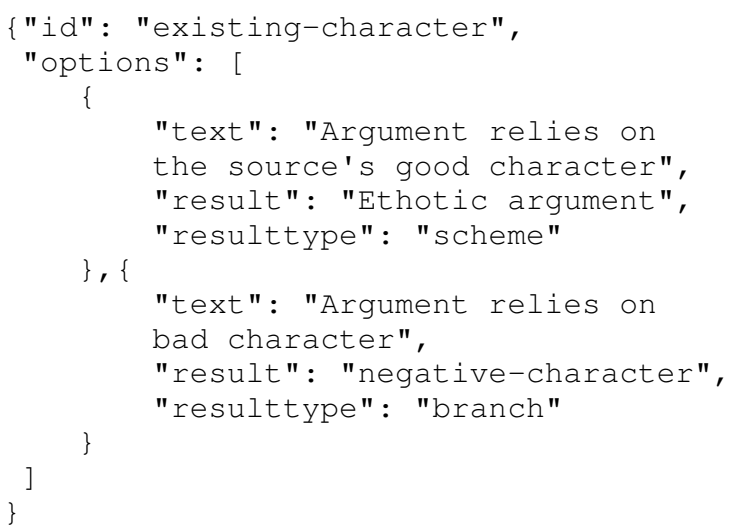

When a user elects to use the ASK to help them select an argument scheme, they are presented with a series of modal dialogue boxes similar to that shown in Figure 3. At each stage, the user selects one of the options and is then either presented with the next dialogue box, or they reach a scheme classification which they can choose to accept and apply. An ordered list of user selections at each stage is recorded so that they can step back through the options if they wish to correct an earlier choice.

\footnotetext{
${ }^{1}$ http://arg.tech/ john/waltonkey.json
} 


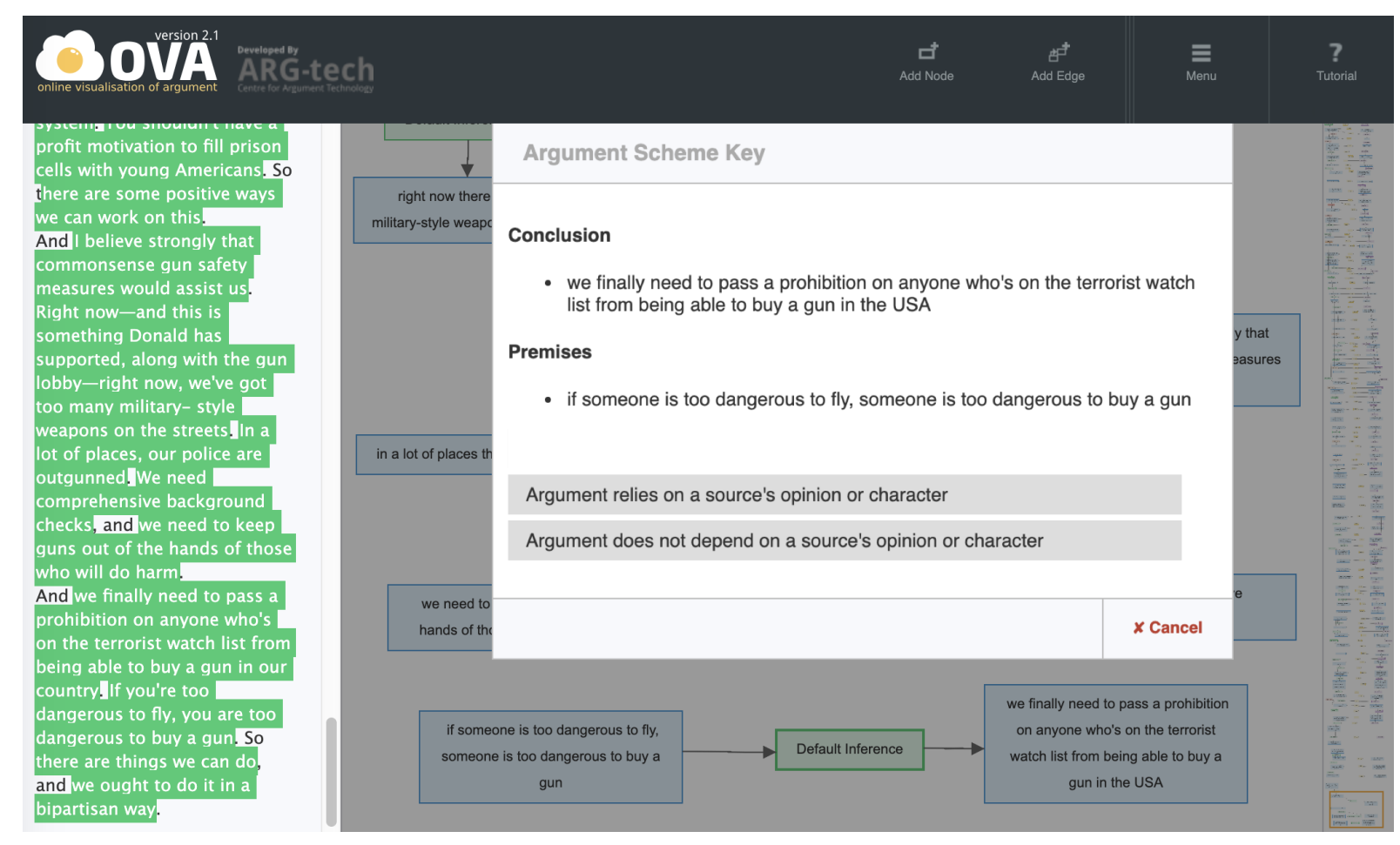

Figure 3: Using the ASK in OVA to annotate the argument scheme used by Clinton in Example (1)

\section{Conclusion}

Identifying the scheme an argument is based on is an important part of evaluating the argumentative quality of discourse. The availability of large, reliable, and representative datasets is essential both to the empirical study of the use of argument schemes in argumentative practice, and to the development of automated classifiers and argument mining techniques. Existing annotated corpora, however, such as those used by Feng and Hirst (2011), and Lawrence and Reed (2015), for the automatic classification of argument schemes, are not validated, of limited size, or do not represent a broad range of scheme types.

Aiming to improve the availability of highquality argument scheme corpora, the online annotation assistant we present here combines a novel annotation method for Walton's typology, with the widely used OVA software for argument analysis. The Argument Scheme Key (ASK) module is available for annotators in OVA at http: / / ova. arg.tech. This work constitutes an intermediate step in the development of automated classifiers, utilising the uniquely identifying characteristics of the ASK. Future work will explore the accuracy and robustness of manual annotations by experts, non-experts, and crowd-sourcing (Musi et al., 2016) using the ASK module in OVA.

\section{Acknowledgments}

This research was supported by the Engineering and Physical Sciences Research Council (EPSRC) in the United Kingdom under grant EP/N014871/1.

\section{References}

Robert Anthony and Mijung Kim. 2015. Challenges and remedies for identifying and classifying argumentation schemes. Argumentation, 29(1):81-113.

Pietro Baroni, Dov Gabbay, Massimiliano Giacomin, and Leendert Van der Torre. 2018. Handbook offormal argumentation, Vol. 1. College Publications.

Carlos Chesñevar, Sanjay Modgil, Iyad Rahwan, Chris Reed, Guillermo Simari, Matthew South, Gerard Vreeswijk, Steven Willmott, et al. 2006. Towards an argument interchange format. The Knowledge Engineering Review, 21(04):293-316.

Jacob Cohen. 1960. A coefficient of agreement for nominal scales. Educational and Psychological Measurement, 20(1):37-46.

Richard A Duschl. 2007. Quality argumentation and epistemic criteria. In Argumentation in science education, pages 159-175. Springer.

F. H. van Eemeren, B. Garssen, E. C. W. Krabbe, A. F. Snoeck Henkemans, B. Verheij, and J. H. M. Wagemans. 2014. Handbook of argumentation theory. Springer. 
F. H. van Eemeren and R. Grootendorst. 1992. Argumentation, communication, and fallacies: $A$ pragma-dialectical perspective. Lawrence Erlbaum Associates.

F. H. van Eemeren, R. Grootendorst, and T. Kruiger. 1978. Argumentatietheorie [Argumentation theory]. Het Spectrum.

Frans H. van Eemeren and Bart Garssen. 2019. Argument schemes: Extending the pragma-dialectical approach. In Proceedings of the 9th Conference of the International Society for the Study of Argumentation (ISSA), pages 308-318, Amsterdam, The Netherlands. Sic Sat.

Vanessa Wei Feng and Graeme Hirst. 2011. Classifying arguments by scheme. In Proceedings of the 49th Annual Meeting of the ACL: Human Language Technologies-Volume 1, pages 987-996. ACL.

Tim van Gelder. 2007. The rationale for rationale. Law, probability and risk, 6(1-4):23-42.

Thomas F Gordon, Henry Prakken, and Douglas Walton. 2007. The carneades model of argument and burden of proof. Artificial Intelligence, 171(10):875-896.

Nancy Green. 2015. Identifying argumentation schemes in genetics research articles. In Proceedings of the 2nd Workshop on Argumentation Mining, pages 12-21, Denver, CO. ACL.

Leo Groarke, Christopher Tindale, and Linda Fisher. 1997. Good reasoning matters! : a constructive approach to critical thinking. Oxford University Press, Toronto.

Randy Allen Harris and Chrysanne Di Marco. 2017. Rhetorical figures, arguments, computation. Argument and Computation, 8(3):211-231.

Arthur C Hastings. 1963. A Reformulation of the Modes of Reasoning in Argumentation. Ph.D. thesis, Northwestern University.

Mathilde Janier, John Lawrence, and Chris Reed. 2014. OVA+: An argument analysis interface. In Proceedings of the Fifth International Conference on Computational Models of Argument (COMMA 2014), pages 463-464, Pitlochry. IOS Press.

M. Kienpointner. 1992. Alltagslogik. Struktur and Funktion von Argumentationsmustern [Everyday logic. Structure and functions of specimens of argumentation]. Fromman-Holzboog.

John Lawrence, Floris Bex, Chris Reed, and Mark Snaith. 2012. AIFdb: Infrastructure for the argument web. In Proceedings of the Fourth COMMA, pages 515-516.

John Lawrence, Joonsuk Park, Katarzyna Budzynska, Claire Cardie, Barbara Konat, and Chris Reed. 2017. Using argumentative structure to interpret debates in online deliberative democracy and erulemaking. ACM Transactions on Internet Technology (TOIT), 17(3):25.

John Lawrence and Chris Reed. 2015. Combining argument mining techniques. In Proceedings of the 2nd Workshop on Argumentation Mining, pages 127-136, Denver, CO. ACL.

Elena Musi, Debanjan Ghosh, and Smaranda Muresan. 2016. Towards feasible guidelines for the annotation of argument schemes. In Proceedings of the $3 \mathrm{rd}$ Workshop on Argumentation Mining, Berlin. ACL.

R. J. Pankhurst. 1978. Biological identification. London: Edward Arnold.

Andreas Peldszus and Manfred Stede. 2013. From argument diagrams to argumentation mining in texts: A survey. International Journal of Cognitive Informatics and Natural Intelligence (IJCINI), 7(1):1-31.

Chaïm Perelman and Lucie Olbrechts-Tyteca. 1969. The New Rhetoric: A Treatise on Argumentation. University of Notre Dame Press.

I. Rahwan and G. R. Simari. 2009. Argumentation in artificial intelligence. Springer.

Chris Reed and Glenn Rowe. 2004. Araucaria: Software for argument analysis, diagramming and representation. International Journal on Artificial Intelligence Tools, 13(4):961-980.

Eddo Rigotti and Sara Greco. 2019. Inference in Argumentation: A Topics-Based Approach to Argument Schemes. Springer.

P. J. Schellens. 1985. Redelijke argumenten. Een onderzoek naar normen voor kritische lezers [Reasonable arguments. A study of norms for critical readers]. Foris.

A. F. Snoeck Henkemans. 1992. Analyzing complex argumentation. SicSat.

Yi Song, Michael Heilman, Beata Beigman Klebanov, and Paul Deane. 2014. Applying argumentation schemes for essay scoring. In Proceedings of the First Workshop on Argumentation Mining, pages 69-78. Association for Computational Linguistics.

Manfred Stede and Jodi Schneider. 2018. Argumentation Mining. Morgan and Claypool Publishers.

Jacky Visser, Barbara Konat, Rory Duthie, Marcin Koszowy, Katarzyna Budzynska, and Chris Reed. 2019a. Argumentation in the 2016 US presidential elections: annotated corpora of television debates and social media reaction. Language Resources and Evaluation.

Jacky Visser, John Lawrence, Jean Wagemans, and Chris Reed. 2018. Revisiting Computational Models of Argument Schemes: Classification, Annotation, Comparison. In Proceedings of the 7th International Conference on Computational Models of 
Argument (COMMA 2018), Warsaw, Poland. IOS Press.

Jacky Visser, John Lawrence, Jean Wagemans, and Chris Reed. 2019b. An annotated corpus of argument schemes in us election debates. In Proceedings of the 9th Conference of the International Society for the Study of Argumentation (ISSA), pages 1101-1111, Amsterdam, The Netherlands. Sic Sat.

E. G. Voss. 1952. The history of keys and phylogenetic trees in systematic biology. Journal of the Science Laboratories, Denison University, 43(1):1-25.

Jean H. M. Wagemans. 2016. Constructing a Periodic Table of Arguments. In Argumentation, Objectivity, and Bias: Proceedings of the 11th International Conference of the Ontario Society for the Study of Argumentation (OSSA), pages 1-12. OSSA.

D. Walton, C. Reed, and F. Macagno. 2008. Argumentation Schemes. Cambridge University Press.

Douglas Walton. 1996. Argumentation schemes for presumptive reasoning. Lawrence Erlbaum Associates, Mahwah, New Jersey.

Douglas Walton. 2006. Fundamentals of critical argumentation. Cambridge University Press.

\section{A ASK: Argument Scheme Key}

1. Argument relies on a source's opinion or character

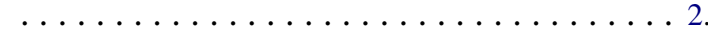

- Argument does not depend on a source's opinion or

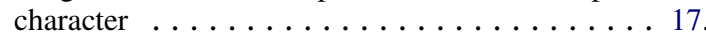

2. Argument is about the source's character ..... 3 .

- Argument is about the source's opinion ...... 9 .

3. Argument establishes the source's character ...... .... Argumentation from interaction of act and person Argument refers to the source's existing character

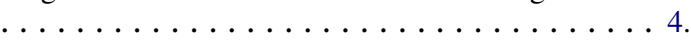

4. Argument relies on the source's good character ..... $\ldots \ldots \ldots \ldots$. . . . . . Ethotic argument Argument relies on bad character ....... 5

5. Source is biased $\ldots \ldots \ldots \ldots \ldots \ldots$

- Argument is not related to bias ......... 7 .

6. Source does not take both sides into account $\ldots \ldots \ldots$

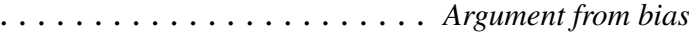

Source's opinion is not acceptable ........... ................. Bias ad hominem

7 (5). Source is of bad overall character . . . . . . .

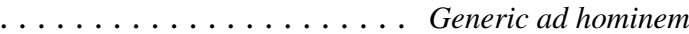

- The source's actions are not compatible with their com-

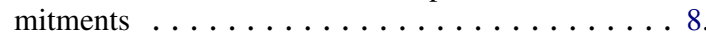

8. Source's actions contradict the advocated position $\ldots$. ................... Pragmatic inconsistency

- Source is not credible due to inconsistent commitments ............... Circumstantial ad hominem

9 (2). Argument establishes a source's opinion . . . 10. Argument is based on an existing opinion ..... 11 .

10. Commitment at issue is consistent with existing commitments . . . . . Argument from commitment

- Commitment at issue is not consistent with existing

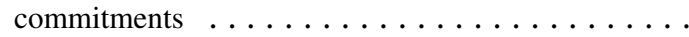
... . . . Argument from inconsistent commitment

11 (9). Source is a general group of people . . . . . . . . ............. Argument from popular opinion
- Source is a specific individual $\ldots \ldots \ldots \ldots$. . . 12 .

12. Source is an expert in the subject domain . . . . . . . . . . . . . . . . Argument from expert opinion

- Source's credibility is not based on domain knowledge

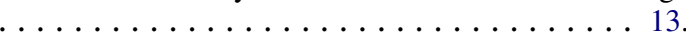

13. Source is a witness $\ldots \ldots \ldots \ldots \ldots$ ... . . . . . Argument from witness testimony

- Source is not a witness ... . . . . . . . . 14 .

14. Argument is based on the source's memories ..... ................. Argument from memory

Argument does not explicitly refer to memories ....

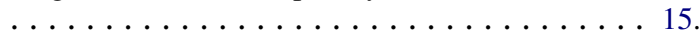

15. Argument is based on the source's visual perception .................. Argument from perception Argument does not explicitly refer to perception ....

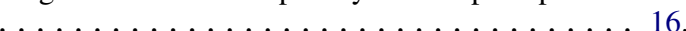

16. Conclusion is about a course of action ......... ........... Two-person practical reasoning Argument is not action-oriented .......... ............. Argument from position to know

17 (1). Conclusion is about a course of action .... 18. Conclusion is not specifically action-oriented . . . 32.

18. Argument focuses on the outcome of an action . . 22 .

- Argument hinges on another motivation for the action ........................... 19

19. Course of action follows an established practice $\ldots .$. ..................... 20.

- Course of action is compared to a similar or alternative

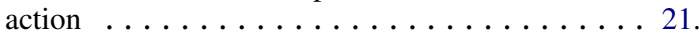

20. Course of action is explicitly regulated $\ldots \ldots \ldots$

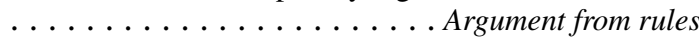
Course of action follows general practices ...... ............. Argument from popular practice

21 (19). Action is best alternative on the basis of prior commitments .......... Argument from sunk costs

- Action is directly compared to another . . . . . . . . . ........... Practical reasoning from analogy

22 (18). Conclusion promotes a positive outcome . . 23. Conclusion prevents a negative outcome . . . . 26.

23. Course of action assists someone else ...... 24 .

- Course of action does not offer help ... . . . 25.

24. Course of action relieves suffering .........

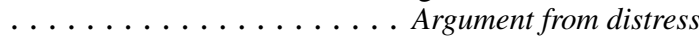
Argument does not mention suffering ......... ............... Argument from need for help

25 (23). Course of action promotes a goal . . . . . . . ........ Argument from (positive) consequences Course of action is not related to an explicit goal ....

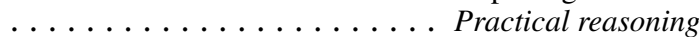

26 (22). Conclusion is in favour of a course of action . . . . ......................... 27 Conclusion is against a course of action . . . . 29.

27. Course of action is already ongoing $\ldots \ldots \ldots \ldots$ ................ Argument from waste Action is still to commence .......... 28 .

28. Action is motivated by fear ............ $\ldots \ldots \ldots \ldots$ Argument from fear appeal - Argument is not about a fearful situation ....... ........ Pragmatic argument from alternatives

29 (26). Chain of events would lead to bad outcome . . . . ............. Slippery slope argument Action's direct outcome is bad ... . . . . . 30.

30. Performing action would lead to punishment $\ldots \ldots$. $\ldots \ldots \ldots \ldots$. . . . . . . . . . . . Argument is not related to specific threat .... 31 .

31. Action would lead to dangerous situation . . . . . . . ............... Argument from danger appeal Action would lead to other bad consequences ..... ......... Argument from (negative) consequences

32 (17). Argument explicitly mentions values . . . . 33. 
- Argument is not specifically value-based .... 34 .

33. Conclusion is about retaining a goal $\ldots \ldots \ldots \ldots$ . . . . . . . . . . . Argument from values

- Conclusion is about valuation . . . . . . . . . . . . . . . . . . . . . . Argumentation from sacrifice

34 (32). Argument is about classification or legal rules

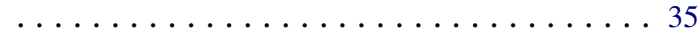

- Argument is not about classification or legal rules ................... 45.

35. Conclusion is about the applicability of a legal rule .................... 36.

- Argument is about meaning or classification . . 39.

36. Chain of similar cases supports application of rule . . . . . . . . . . . . . Precedent slippery slope argument Rule does not apply in this case ........ 37

37. Rule does not apply due to special circumstances .... . . . . . . . . . . Argument from plea for excuse Argument is about an exception to the rule . . . 38 .

38. Premise refers to a recognised type of exception .... . . . . . . . . . Argument for an exceptional case

- Conclusion establishes a new exception to the rule . . . . . . . . . Argument from precedent

39 (35). Argument is based on an existing meaning or classification .................440.

- Argument establishes a new meaning or classification .................... 41

40. Argument uses a term with arbitrary meaning . . . . . . - Argument from arbitrariness of a verbal classification

- Argument uses a term with vague meaning . . . . . . . ... Argument from vagueness of a verbal classification

41 (39). Argument relies on a chain of reasoning . . . 42.

- Argument constitutes a single reasoning step . . 43.

42. Conclusion establishes a new classification ....... . . . . . . . . . . Sorites slippery slope argument

- Conclusion rejects a new classification . . . . . . . . . . . . . . . . . . Verbal slippery slope argument

43 (41). Conclusion establishes a new meaning . . . . . . . . . . . . . Argument for constitutive-rule claims Conclusion establishes a new classification ... . 44.

44. New classification is based on a definition ....... . . . . Argument from definition to verbal classification

- New classification is based on a property ....... . . . . . . . . . . Argument from verbal classification

45 (34). Argument relies on a chain of reasoning ... 46.

- Argument constitutes a single reasoning step . . . 47.

46. Conclusion is about accepting something ....... . . . . . . . . . . A Argument from gradualism

- Conclusion is about rejecting something . . . . . . . . . . . . . . . . . Full slippery slope argument

47 (45). Argument relies on a causal relation ... . 48.

- Argument does not specifically rely on causality .... . . . . . . . . . . . . . . . . . . 49

48. Argument relies on a known causal relation ...... . . . . . . . . . Argument from cause to effect

- Argument establishes a new causal relation ....... . . . . . . . . . . Argument from correlation to cause

49 (47). Argument relies on an individual case . . . 54

- Argument relates to (a collection of) other cases . . . .

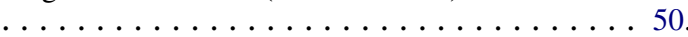

50. Conclusion attributes a property $\ldots \ldots \ldots \ldots 5$.

- Conclusion rejects a property . . . . . . . . 53

51. Argument is based on group membership . . . . . . . .... . Argumentation from the group and its members

Argument is based on a part-whole relation . . . . 52.

52. Reasoning goes from the parts to the whole ...... . . . . . . . . . . Argument from composition Reasoning goes from the whole to its parts ...... . . . . . . . . . . . . . Argument from division

53 (50). Argument is based on contradictory properties . . . . . . . . . . Argument from oppositions
- Subject is not an element of the expected class ..... . . . . . . . . . Epistemic argument from ignorance 54 (49). Argument refers to a characteristic sign . . 55. Argument is based on a comparison . . . . . . 57

55. Characteristic sign is absent $\ldots \ldots \ldots \ldots \ldots \ldots$ .............. Argument from ignorance Characteristic sign is present $\ldots \ldots \ldots \ldots 56$

56. Conclusion is a hypothesis $\ldots \ldots \ldots \ldots \ldots$ . . . . . . . Argument from evidence to a hypothesis Premise mentions a sign . . . . . Argument from sign

57 (54). Case at issue is similar to compared case(s) . . . . .................... 58

- Case at issue is different from compared case(s) . . . . ....................... 59

58. Argument compares between particular instances .... . . . . . . . . . . . . Argument from analogy

- Argument generalises from a particular instance .... . . . . . . . . . . . Argument from example

59 (57). Explanation is better than the alternatives .... . . . . . . . . . . Abductive argumentation scheme Argument compares to an opposite case . . . . 60.

60. Argument rejects an alternative $\ldots \ldots \ldots \ldots \ldots$ . . . . . . . . A Argument from alternatives Argument based on opposite treatment in a contrary case ...... Rhetorical argument from oppositions 